Received: 13 December 2018

Accepted: 13 February 2019

Published online: 05 March 2019

\section{OPEN A novel echocardiographic method closely agrees with cardiac magnetic resonance in the assessment of left ventricular function in infarcted mice}

\author{
Ilaria Russo $\mathbb{1}^{1}$, Edoardo Micotti ${ }^{2}$, Francesca Fumagalli ${ }^{1}$, Michela Magnoli ${ }^{1}$, \\ Giuseppe Ristagno ${ }^{1}$, Roberto Latini $^{1}$ \& Lidia Staszewsky ${ }^{1}$
}

Cardiac Magnetic Resonance (CMR) is the gold standard for left ventricular (LV) function assessment in small rodents and, though echocardiography (ECHO) has been proposed as an alternative method, LV volumes may be underestimated when marked eccentric remodeling is present. In the present study we described a novel echocardiographic method and we tested the agreement with CMR for LV volumes and ejection fraction calculation in mice with experimental myocardial infarction. Sham-operated and infarcted mice, subjected to Coronary Artery Ligation, underwent ECHO and CMR. Volumes and ejection fraction were calculated by ECHO using a standard Simpson's modified method (ECHO pLAX) or a method from sequential parasternal short axis (ECHO pSAX) acquired mechanically by translating the probe every $1 \mathrm{~mm}$ along the left ventricle. The mean differences \pm 1.96 standard deviation near to zero suggested close agreement between ECHO pSAX and CMR; contrarily ECHO pLAX agreement with CMR was lower. In addition, ECHO was three times shorter and cheaper (Relative cost difference: pLAX: $-66 \%$ and pSAX - 57\%) than CMR. In conclusion, ECHO pSAX is a new, fast, cheap and accurate method for LV function assessment in mice.

Wild-type and genetically manipulated mice are widely used in experimental models of myocardial infarction to examine the characteristics of cardiac phenotype, the role of a specific protein, or to test in vivo the efficacy of new therapeutic approaches ${ }^{1}$. The functional response of the murine heart to any intervention can now be studied in vivo using several non-invasive imaging techniques ${ }^{2}$. To date, the two most widely used imaging methods are echocardiography (ECHO) and Cardiac Magnetic Resonance (CMR).

Echocardiography is a simple, fast, cheap and safe imaging method ${ }^{3}$. In the last two decades, it has been the most frequently used technique to assess left ventricular (LV) structure and function in mice. New high-resolution instruments have now been designed, specifically to study small rodents, using high-frequency probes $(30-50 \mathrm{MHz})$ with higher spatial resolution $(30 \mu \mathrm{m})$, allowing more accurate assessment of the left ventri$\mathrm{cle}^{2,3}$. However, the main limits of this method are its geometric assumptions, the acoustic window characteristics, probe positioning errors, and a highly subjective approach that makes a skilled sonographer indispensable ${ }^{3}$. Moreover, despite echocardiography is the routine imaging method used for non-invasive left ventricular function assessment in small rodents, a systematic critical evaluation of imaging quality and of the resulted data, is often lacking.

Cardiac Magnetic Resonance is the gold standard for non-invasive assessment of cardiac function in small rodents $^{4-6}$. With the strength of its significant intrinsic contrast and not dependent on geometric assumptions, it allows the acquisition of contiguous slices of short-axis images along the entire length of the left ventricle, permitting accurate estimates of LV volumes (LVVs). Although CMR is the most reliable method, with the highest

${ }^{1}$ Department of Cardiovascular Research, Mario Negri Institute for Pharmacological Research IRCCS, Milan, Italy. ${ }^{2}$ Department of Neuroscience, Mario Negri Institute for Pharmacological Research IRCCS, Milan, Italy. Correspondence and requests for materials should be addressed to I.R. (email: russilar9@gmail.com) 
sensitivity and specificity to study the heart anatomy in vivo, it is expensive and fairly time-consuming compared to ECHO, which why it is not routinely used in large series of animals or for serially repeated exams ${ }^{4-6}$.

Several studies have proposed new ECHO approaches for assessing cardiac function, in order to avoid the limits of the standard left chamber quantification in small rodents ${ }^{4,7-9}$. One of these methods is the echocardiographic 3D reconstruction of the left ventricle ${ }^{4}$, which has been found to have good agreement with CMR for Left Ventricular Ejection Fraction (LVEF) calculation in several clinical studies ${ }^{10}$. However, it is not widely used, and is still not available in most of the experimental laboratories.

Another general crucial issue is that stringent and robust comparisons between ECHO and CMR in the setting of pharmacological preclinical research not specialized in imaging are still necessary.

Here we investigated whether an echocardiographic approach which allows acquiring serial short-axis images using a high-resolution ultrasound system allows a better agreement with CMR for LV function assessment in a mouse model of myocardial infarction.

\section{Methods}

All supporting data are available within the article and upon reasonable request to the corresponding author.

Animal use and care. Mice were housed at constant room temperature of $23^{\circ} \mathrm{C}$ and relative humidity $(60 \pm 5 \%)$ with ad libitum access to food and water and fixed 12 hours light/dark cycle. Procedures involving animals and their care were conducted in conformity with the institutional guidelines at the Mario Negri Institute for Pharmacological Research IRCCS (Istituto di Ricovero e Cura a Carattere Scientifico) in compliance with national (D.lgs 26/2014; Authorization no. 19/2008-A issued March 6, 2008 by the Ministry of Health of Italy) and international (European Economic Community Council Directive 2010/63/UE; the National Institutes of Health Guide for the Care and Use of Laboratory Animals, 2011 edition) laws and policies. Procedures were reviewed and approved by the Mario Negri Institute Animal Care and Use Committee, which includes ad hoc members for ethical issues, and by the Italian Ministry of Health (Decreto no. 62/2012-B). Animal facilities meet international standards and are regularly checked by a certified veterinarian who is responsible for health monitoring, animal welfare supervision, experimental protocols, and review of procedures.

Surgical procedures. A total of $25 \mathrm{C} 57 \mathrm{BL} / 6 \mathrm{~J}$ mice (body weight ranging 23-31 g) were anesthetized with isoflurane and permanent occlusion was induced by Coronary Artery Ligation (CAL), as previously described ${ }^{11}$. In 16 sham-operated mice (SHAM) surgery was done without CAL with the suture placed loosely around the left coronary artery. All animals received buprenorphine $(0.1 \mathrm{mg} / \mathrm{kg}$ s.c. $\mathrm{q} 12 \mathrm{~h})$ for one day as post-surgical analgesia and were followed for six weeks. Mortality was constantly recorded until six weeks after surgery. Mortality rate in CAL mice was $28 \%(n=18$ survived) while all SHAM mice $(n=16)$ reached the end of the follow up.

ECHO: Image acquisition. Echocardiography was performed using a $30 \mathrm{MHz}$ mechanical probe with a spatial resolution of $30 \mu \mathrm{m}$ (VisualSonics, Vevo 770, Toronto, Canada) 6 weeks after CAL on mice anesthetized with isoflurane $\left(0.5-1.5 \%\right.$ in $\left.\mathrm{O}_{2}\right)$. Animals were positioned on a rail system for maintenance of the body temperature $\left(37^{\circ} \mathrm{C} \pm 0.5^{\circ} \mathrm{C}\right)$ and the probe position under electrocardiographic (EKG) and respiratory monitoring for the entire duration of the exam.

Parasternal long-axis (pLAX) B-mode image was acquired, optimizing the LV length for LV volume measurements and ejection fraction calculations (Fig. 1A). Parasternal short-axis (pSAX) 2D images of the left ventricle were recorded by translating the probe every $1 \mathrm{~mm}$ from the base to the apex (Fig. 1B). Cine loops containing five cardiac cycles (25-35 frames per cardiac cycle at a frame rate of 300-400/sec) were stored in Digital Imaging and COmmunications in Medicine (DICOM) format and measurements were taken off-line.

ECHO: LV volumes and ejection fraction calculation. Measurements were made off line by two sonographers (I.R., L.S.) blinded to experimental groups. LV endocardial areas were drawn manually in end-diastolic and end-systolic frames in images from pLAX and pSAX views. LV end-diastolic and end-systolic volumes (LVEDV and LVESV) were calculated using two methods: (1) a standard Single-plane modified Simpson (ComPACS software, Medimatic S.R.L, Genoa, Italy) from the pLAX view (ECHO pLAX, Fig. 2A); (2) from the pSAX view acquiring sequential short-axis $1 \mathrm{~mm}$-thickness slices covering the entire left ventricle (ECHO pSAX, Fig. 2B). Diastolic and systolic endocardial areas were drawn manually in each slice and volumes were calculated with the formula used in CMR ${ }^{12,13}$ as follows: $\Sigma$ (LVEDA) or $\Sigma$ (LVESA)*slices thickness (LVEDA, end-diastolic area; LVESA, end-systolic area). Ejection fraction was calculated following the formula: (LVEDV-LVESV)/ $\operatorname{LVEDV}^{*} 100$. For each parameter, the mean of 3-5 consecutive measurements was calculated.

CMR: Image acquisition. Cardiac Magnetic Resonance was done three days after ECHO on a horizontal bore 7 Tesla USR preclinical MRI system (BioSpec 70/30, BrukerBioSpin, Germany) with a shielded gradient insert (BGA 12, 400mT/m; rise time $110 \mathrm{~ms}$ ). A linear volume coil and anatomically shaped surface coil were used to transmit/receive the magnetic resonance signals (linear volume coil inner diameter, $72 \mathrm{~mm}$; surface coil inner diameter, $10 \mathrm{~mm}$, Bruker BioSpin, Germany). After the localizer, scans (segmented double-gated FLASH imaging) were run to ensure correct positioning, tuning and matching the probe; slice-selective shimming and flip angle calibration were done manually before each experiment. Cine-MRI pulse-sequences were: matrix $256 \times 128$; FOV $40.0 \times 20.0 \mathrm{~mm}$; echo time, $2.98 \mathrm{~ms}$; flip angle $15^{\circ}$; slice thickness, $1 \mathrm{~mm}$; number of averages, 6 ; repetition time, $12 \mathrm{~ms}$.

Mice, anesthetized by facemask with isoflurane (at the same concentration used in ECHO) and $0.3 \mathrm{~L} / \mathrm{min}_{2}$, were positioned in a purpose-built cradle and EKG electrodes were attached to the front paws. A pressure transducer for respiratory gating was positioned above the abdomen. A fiber optic probe was used to monitor the rectal temperature. EKG and respiratory signals were processed and displayed using a gating device and temperature 
A

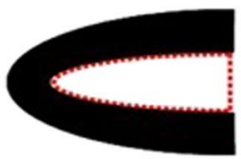

END-DIASTOLE

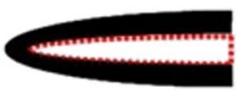

END-SYSTOLE
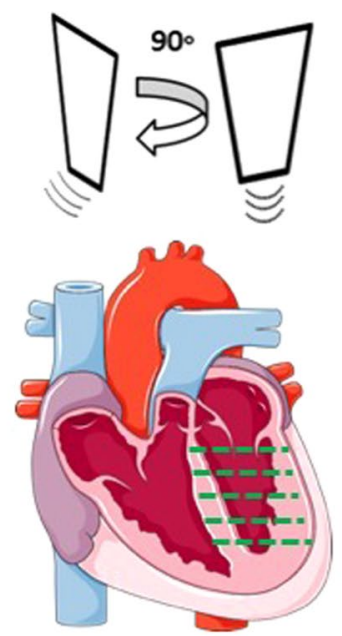

APEX

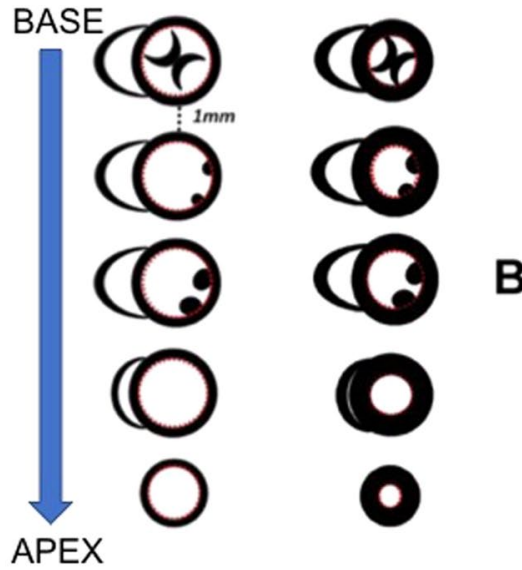

END-DIASTOLE END-SYSTOLE

Figure 1. ECHO pLAX and ECHO pSAX methods. Schematic representation of ECHO pLAX (A) and ECHO pSAX (B) acquisition. (A) 2D ECHO cine-loops were taken from parasternal long axis view and endocardial diastolic and systolic areas were traced according to the maximal and minimal LV expansion. (B) transducer was rotated approximately 90 degrees clockwise from the parasternal long axis until left ventricle appeared circular. Serial cine-loops of 2D images from parasternal short axis view were acquired from base to apex by translating the ultrasound probe by $1 \mathrm{~mm}$ using a customized system. The figure was drawn using Servier Medical Art (https://smart.servier.com).

was maintained at $37 \pm 0.5^{\circ} \mathrm{C}$ throughout the examination (SA Instruments, Stony Brook, NY Model 1025 MRI compatible physiological monitoring system).

After the image plane orientation from the coronal, sagittal and axial LV long-axis, a vertical long axis (VLA) was obtained and, orthogonal to it, adjusting orientation from a short axis slice at the mid-papillary muscle level, a horizontal long axis (HLA) was acquired followed by $1 \mathrm{~mm}$ serial short-axis slices covering the entire LV length (Fig. 2C). Six to eleven short-axis slices were acquired from base to apex. Sixteen frames per slice for one cine sequence were stored (two cardiac cycles) to ensure the acquisition of maximum and minimum LV expansion (end-diastole and end-systole). Images were exported in DICOM format and analyzed off-line.

CMR: LV volumes and ejection fraction calculation. Two investigators (I.R., L.S.) blinded to the experimental conditions analyzed CMR recordings. End-diastolic and end-systolic parameters were measured in selected frames respectively according to the visual estimation of the maximal and minimal ventricular cavity. End-diastolic and end-systolic endocardial areas of each slice were traced manually. LV volumes and ejection fraction were calculated using the CMR formula.

All echocardiographic and CMR examinations were included in the analysis if they presented at least five distinct sequential short-axis slices where epicardial and endocardial borders were adequately visualized.

In vivo measurement of infarct size. Infarct size was calculated in vivo by CMR considering the areas of akinesis. In the end-diastolic frames, the epicardial and endocardial circumferences were traced separately from the length of the infarcted tissue ${ }^{5}$. Three to five short-axis views were considered from the first slice that showed an akinetic segment to the last one to obtain a measurement of infarct size applying the formula: $\Sigma\left(\mathrm{I}_{\text {epi }} /\right.$ $\left.\left.\mathrm{T}_{\text {epi }}+\mathrm{I}_{\text {endo }} / \mathrm{T}_{\text {endo }}\right) * 100\right] / \mathrm{n}$ slices $\left(\mathrm{T}_{\text {epi }}\right.$ and $\mathrm{T}_{\text {endo }}=$ total epicardial and endocardial circumference of left ventricle; $\mathrm{I}_{\text {epi }}$ and $\mathrm{I}_{\text {endo }}=$ epicardial and endocardial length of infarcted tissue). Infarct size is expressed as percentage of the left ventricle.

Sample size calculation and statistical analysis. For sample size calculation, we assumed that the mean difference $(\mu)$, the standard deviation of difference $(\sigma)$ and the predefined clinical limits of agreement $(\delta)$ between ECHO and CMR in LVEF values should not have been greater than $-0.3 \pm 8.2 \%$, with $95 \%$ limits of agreement between -16.4 and 15.7; these values are based on our experience and are also considered clinically plausible ${ }^{10}$. According to the above statement, we calculated an approximate sample size of 19 mice per group in order to apply the Bland and Altman method with a non-central t-distribution assuming a two-side $\alpha$ error of $0.05 \%$ and a power of $80 \%{ }^{14}$. In addition, considering a six weeks mortality rate of $30 \%$ in CAL mice, the number of animals to include in the CAL group was 25. 
ECHO

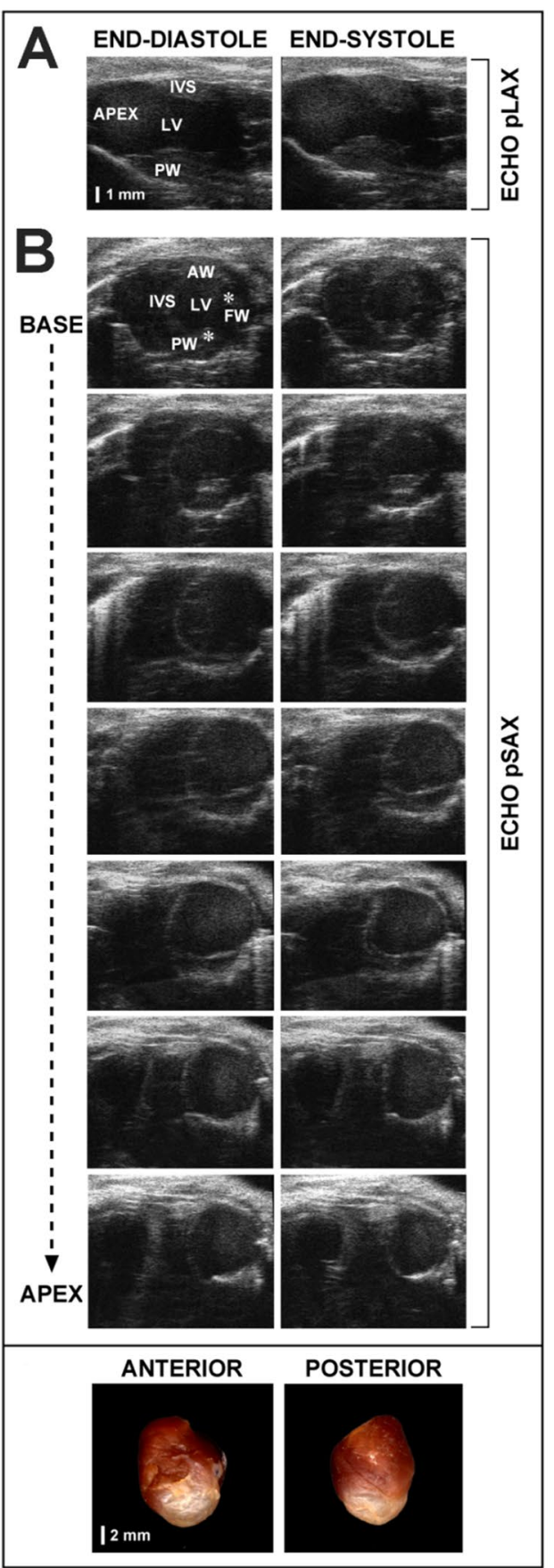

CMR

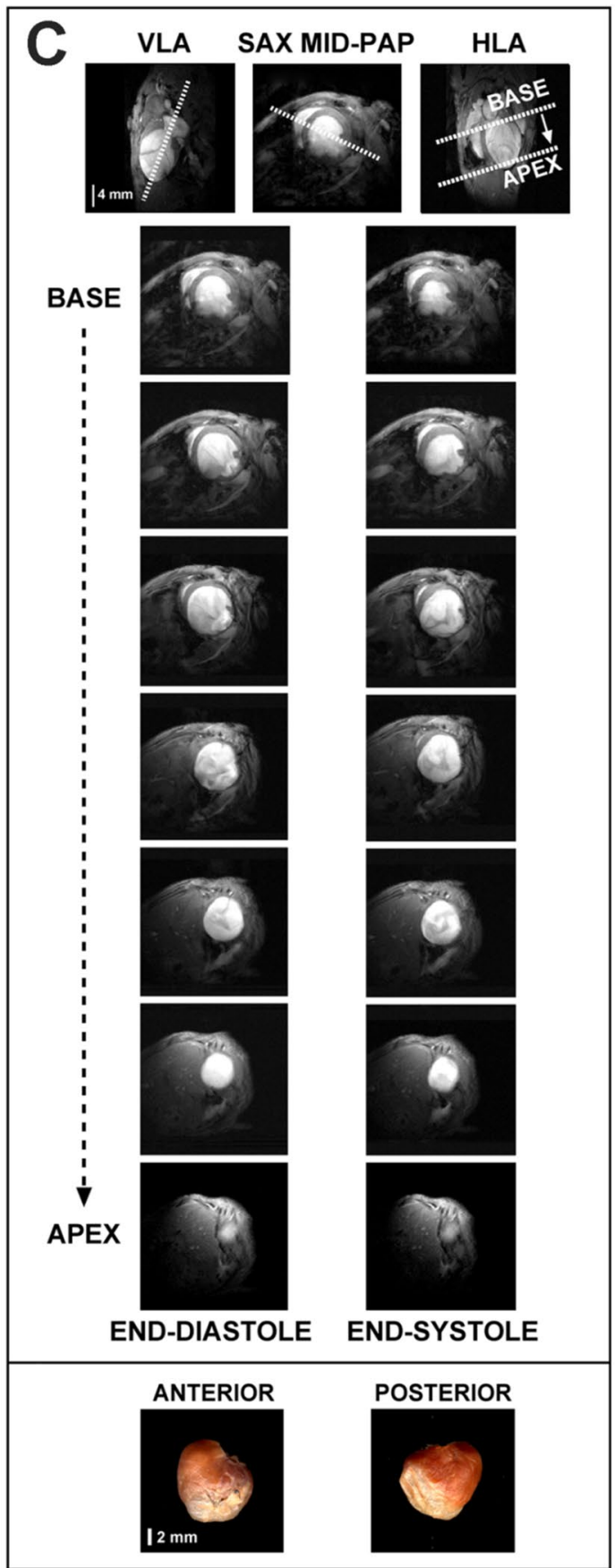

Figure 2. ECHO and CMR images. In vivo echocardiographic images of infarcted heart taken from 2D parasternal long axis (A) and from serial consecutive parasternal short axis (B) and used to apply the ECHO pLAX and the ECHO pSAX methods, respectively. Both echocardiographic methods were used to calculate LV volumes and ejection fraction and were compared to CMR $(\mathbf{C})$. Corresponding ex-vivo photographs of anterior and posterior walls of the left ventricle in corresponding animals are presented in the bottom of the panel. AW, anterior wall; FW, free wall; IVS, interventricular septum; LV, left ventricle; PW, posterior wall; HLA, horizontal long axis; MID-PAP, mid-papillary level; VLA, vertical long axis. *Indicates papillary muscles.

Data are expressed as mean \pm standard error (SE). Differences between two groups were analyzed for statistical significance with an unpaired Student's t-test. Correlations $(r)$ between CMR and echocardiographic measurements and between infarct size and LV volumes and ejection fraction measured by ECHO and CMR were tested by Pearson's method. Limits of agreement between imaging methods were established as the mean difference (bias) \pm 1.96 standard deviation (SD) of the differences, as described by Bland and Altman ${ }^{15,16}$. We calculated $95 \%$ 


\begin{tabular}{|l|l|l|}
\hline & SHAM & CAL \\
\hline $\mathrm{n}$ & 16 & 18 \\
\hline BW $(\mathrm{g})$ & $26.2 \pm 0.8$ & $26.6 \pm 0.5$ \\
\hline HW $(\mathrm{mg})$ & $109.4 \pm 3.0$ & $137.9 \pm 4.4^{\#}$ \\
\hline HW/BW & $4.2 \pm 0.1$ & $5.2 \pm 0.5^{\Im}$ \\
\hline Infarct size (\%) & & $40.2 \pm 3.3$ \\
\hline
\end{tabular}

Table 1. In vivo data. Abbreviations: CAL, Coronary Artery Ligation; n, number of animals; BW, body weight; $\mathrm{HW}$, heart weight. P value from Student t-test: ${ }^{\#} \mathrm{p}<0.0001$ vs. SHAM; ${ }^{\S} \mathrm{p}<0.001$ vs. SHAM.

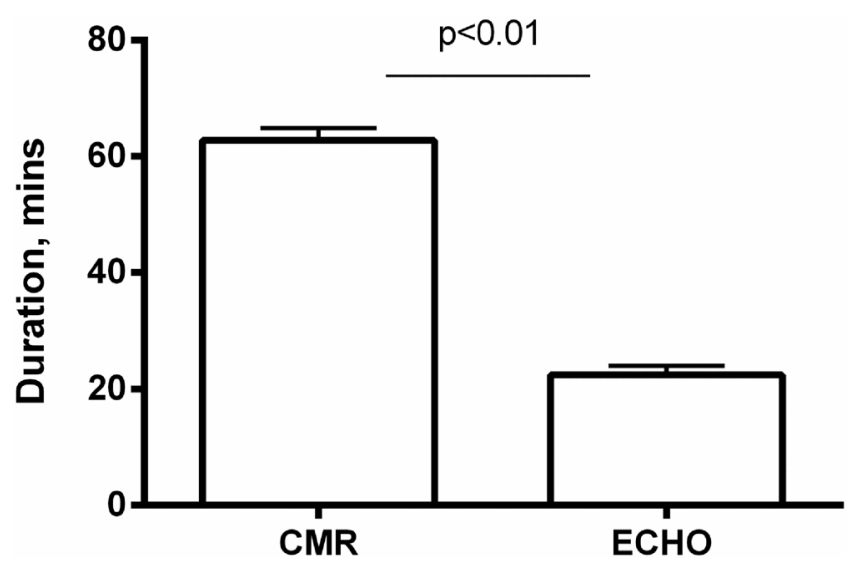

Figure 3. Acquisition time. Comparison between ECHO and CMR imaging duration considering all 34 animals. Data are expressed as mean \pm SE. P value from Student's t-test.

limits of agreement for means and mean differences as $1.96 *$ SD. Statistical significance was set at 5\% (Graph Pad Prism 6.0).

The reproducibility of ECHO pLAX, ECHO pSAX and CMR for LV volumes and ejection fraction measurements was evaluated by calculating the intra-observer and inter-observer variability in both SHAM and CAL groups. Variability was defined as the absolute difference between the corresponding repeated measurements for each animal and expressed as percentage of their mean as represented in the following formula ${ }^{17,18}$ : $\%$ variability $=($ measure 1 -measure 2 /average of measure 1 and measure 2$) * 100$. The resulted data are expressed as mean \pm SD.

\section{Results}

In vivo data. Six weeks after surgery body weight (BW) was not different in CAL and SHAM mice (Table 1). Although CMR total acquisition time was three-times longer than ECHO (CMR 68 mins vs. ECHO 23 mins, Fig. 3), the duration of the CMR and ECHO exams was similar in SHAM and CAL mice (CMR: 60 mins and 65 mins, ECHO: 23 mins and 22 mins, respectively). The heart rate did not differ either between the two groups (Table 2).

In mice with CAL, infarct size ranged between $11.4 \%$ and $69.1 \%$ (mean value $=40.2 \pm 3.3 \%$, Table 1 ), LVEDV was 2.2 times greater in CAL compared with SHAM mice $(\mathrm{p}<0.05)$, LVESV resulted 5.0 times greater and LVEF was 2.2 -fold lower $(\mathrm{p}<0.05)$. Mean values of LV parameters studied are presented in Table 2.

Heart weight and the ratio of heart weight to body weight were significantly higher in CAL than SHAM mice $(+27 \%, \mathrm{p}<0.0001$ and $+24 \%, \mathrm{p}<0.001$, $)$ (Table 1$)$.

Agreement between ECHO and CMR. In SHAM mice, the mean of differences between ECHO pLAX and CMR and ECHO pSAX and CMR for LVVs and LVEF was close to zero (Fig. 4A,B and Table 3). The smaller range of $95 \%$ limits of agreement when ECHO pLAX (-3.2-16.8\%) was compared to CMR indicated that this method was more accurate than ECHO pSAX (-20.6-14.0\%) for LVEF calculation in SHAM mice (Fig. 4A,B).

In CAL mice, ECHO pLAX underestimated LVVs (Fig. 4C), particularly LVESV (mean difference \pm SD $11.5 \pm 38.22 \mu \mathrm{L}$, Table 3). When ECHO pSAX was compared to CMR, the mean differences were close to zero for all studied parameters (Table 3 ) and the $95 \%$ limits of agreement showed a consistently smaller variation of the differences (Fig. 4D).

Correlation between CMR and ECHO. As shown in Supplementary Fig. 1, in SHAM mice the correlation between LVVs and LVEF measured by ECHO and those obtained by CMR was not significant. In contrast, in CAL animals there was a significant linear correlation between LVVs and LVEF calculated by CMR with the values given by each of the ECHO methods used in the study. 


\begin{tabular}{|l|l|l|l|}
\hline & SHAM & CAL & P values \\
\hline \multicolumn{5}{|l|}{ HR $($ bpm $)$} & $493 \pm 12$ & $463 \pm 15$ & NS \\
\hline CMR & $460 \pm 19$ & $444 \pm 16$ & NS \\
\hline ECHO & $48.6 \pm 6.0$ & $108.1 \pm 12.3$ & $<0.01$ \\
\hline LVEDV $(\boldsymbol{\mu l})$ & $48.9 \pm 1.7$ & $102.3 \pm 5.3$ & $<0.01$ \\
\hline CMR & $46.4 \pm 2.4$ & $112.0 \pm 12$ & $<0.01$ \\
\hline ECHO pLAX & $16.0 \pm 0.7$ & $79.3 \pm 11.6$ & $<0.01$ \\
\hline ECHO pSAX & $12.9 \pm 1.0$ & $67.8 \pm 4.8$ & $<0.01$ \\
\hline LVESV $(\boldsymbol{\mu l})$ & $16.4 \pm 1.3$ & $82.0 \pm 11.4$ & $<0.01$ \\
\hline CMR & $67.1 \pm 1.0$ & $30.0 \pm 2.8$ & $<0.01$ \\
\hline ECHO pLAX & $73.9 \pm 1.4$ & $33.9 \pm 2.1$ & $<0.01$ \\
\hline ECHO pSAX & $63.6 \pm 2.2$ & $29.8 \pm 2.3$ & $<0.01$ \\
\hline LVEF $(\%)$ & CMR &
\end{tabular}

Table 2. CMR and ECHO parameters in SHAM and CAL mice. Data are shown as mean \pm SE; Abbreviations: bpm, beats per minute. LVEDV, left ventricular end-diastolic volume; LVESV, left ventricular end-systolic volume; LVEF, left ventricular ejection fraction. P values from Student's t-test.

Inter- and intra-observer variability. The inter-observer and intra-observer variability in LVEDV, LVESV and LVEF calculation in SHAM and CAL mice for all studied imaging methods (Table 4) showed that all three methods are reproducible. In SHAM mice, a slightly higher percentage of inter-observer variability was observed for LVESV measurements in CMR and ECHO pLAX. In the CAL group, only small differences between observers were found and were higher for LVEDV measurements in CMR and ECHO pSAX and for LVESV in CMR.

\section{Discussion}

In the present study we have showed a close agreement between an accessible and fast echocardiographic method, the ECHO pSAX, and the gold standard CMR for LV volumes and ejection fraction calculation in mice with myocardial infarction. This finding has valuable implications in the experimental setting, particularly for longitudinal studies and animal welfare. In mice with a non-asymmetric left ventricle, ECHO pLAX measurements agree closely with those of CMR, thus this standard ECHO method might be confirmed as a fast and accurate approach for assessing systolic function in non-infarcted left ventricles.

This study showed, for the first time to our knowledge, the accuracy of a new high-resolution echocardiographic method of simple manual use for LV volumes and ejection fraction calculation in a large number of mice with myocardial infarction. Our data confirm previous results obtained by two independent studies with smaller number of mice and the use of an echocardiographic machine equipped with a $7 / 15 \mathrm{MHz}$ linear-array probe ${ }^{4,7}$.

Echocardiography with high spatial resolution $(30 \mu \mathrm{m})$ along with an ad hoc instrument for LV scanning allowed contiguous cross-sectional segmentation of the left ventricle and more appropriate comparison between ECHO and CMR (Figs 1 and 2)

A customized hardware/software interface was developed in the last generation of echocardiographic machines in order to control the motor stage holding the ultrasound transducer and trigger the scanner to acquire and store data. This tool allowed for the automatic acquisition of finely sampled 2D short-axis loops providing for a 3D assessment of the left ventricle.

As well known, the quantification of LV remodeling through LV volumes and ejection fraction measurements is useful for assessing the severity of LV injury, establishing the prognosis ${ }^{7}$ and studying the response to a specific treatment. Even if it is widely accepted that in an asymmetric left ventricle LV volumes and ejection fraction calculation is less accurate if derived from M-mode or from 2D long-axis view, these methods are the most frequently and routinely used in experimental settings.

Recently a 3D echocardiographic method has been published with a customized hardware/software interface developed to control a motor stage that holds the ultrasound transducer and trigger the scanner for the acquisition of serial sampled 2D short-axis loops. This method was used to measure LV volumes and ejection fraction in mice with myocardial infarction but, to our knowledge, any correlation data and/or agreement was provided with CMR, the gold standard method to evaluate LV function ${ }^{19}$.

A recent study ${ }^{20}$ has compared ECHO and CMR in CAL mice by using echocardiographic serial slices obtained from parasternal long axis view. Interestingly the results showed an agreement in LV volumes and ejection fraction measurements between the studied methods which was similar to what has been found in our study, particularly for ejection fraction calculation, using the ECHO pLAX method (mean difference: $3.4 \%$ vs. 3.8\%; $95 \%$ limits of agreement: $-16.9-23.6$ vs. $-17.5-25.1$, Fig. 4). However the agreement observed with ECHO pSAX in our study was closer (mean difference: $-0.3 \%$; $95 \%$ limits of agreement: $-16-16$, Fig. 4 ).

Our validation suggests that ECHO pLAX can be proposed as the elective method for LV volumes and ejection fraction calculation in symmetrical ventricles. LV ejection fraction in mice was slightly higher by ECHO pLAX compared to CMR (mean difference \pm SD: $6.8 \pm 5.1 \%$, Table 3 ). This might be ascribed to long-axis peak velocities during systole which are slightly higher and rather homogeneous distributed in LV segments in mice 

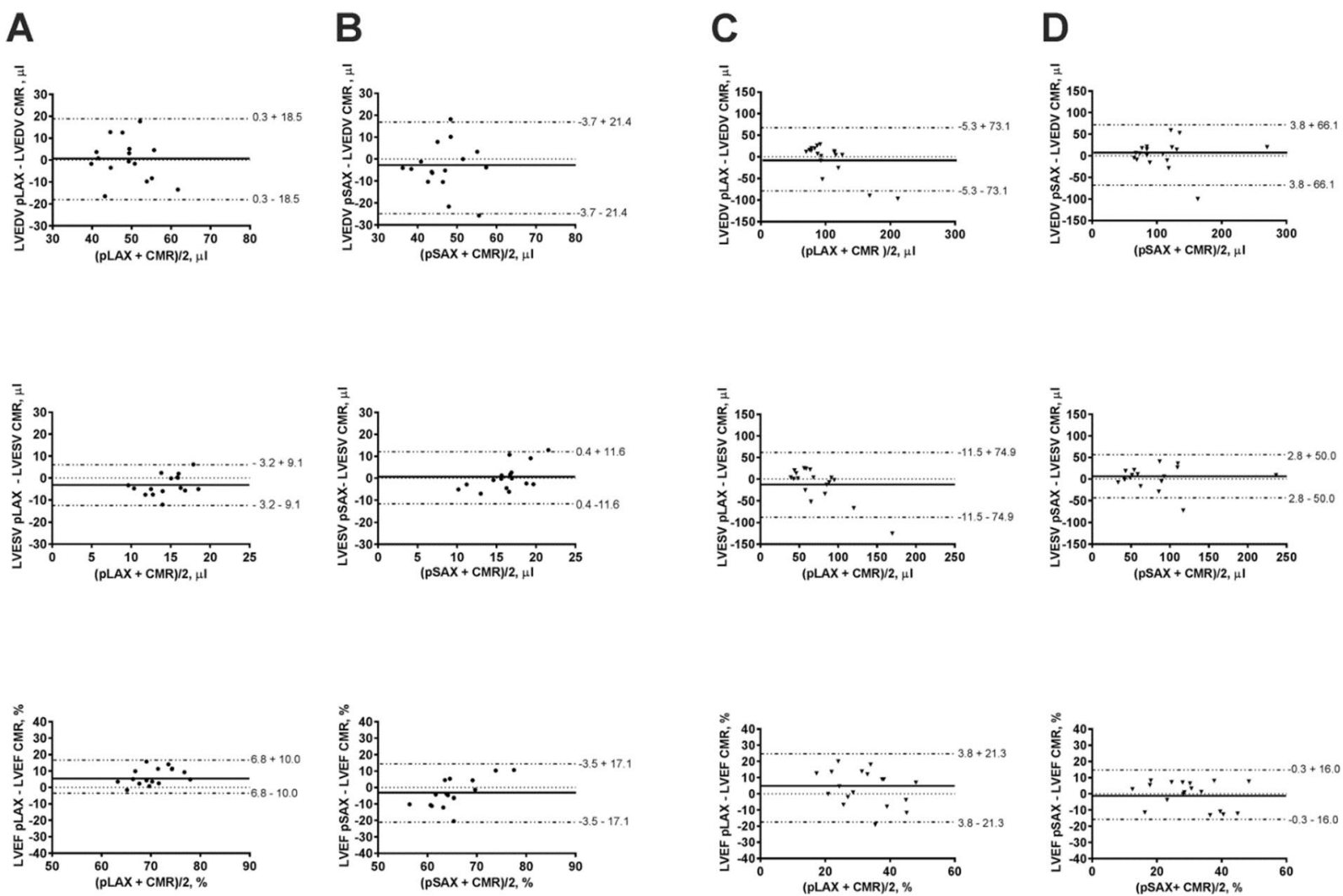

\section{SHAM}

\section{CAL}

Figure 4. Bland and Altman plots for SHAM and CAL mice. The agreements between CMR and ECHO pLAX $(\mathbf{A}, \mathbf{C})$ and between CMR and ECHO pSAX $(\mathbf{B}, \mathbf{D})$ in SHAM and CAL mice are presented. See corresponding Table 3 for each mean difference. Single CMR and ECHO parameters are presented in Table 2.

\begin{tabular}{|l|c|c|c|c|}
\hline \multirow{2}{*}{} & \multicolumn{2}{|l|}{ SHAM } & \multicolumn{2}{l|}{ CAL } \\
\cline { 2 - 5 } & ECHO pLAX & ECHO pSAX & ECHO pLAX & ECHO pSAX \\
\hline LVEDV $(\mu \mathrm{l})$ & $-0.3 \pm 9.4$ & $-3.7 \pm 10.9$ & $-5.3 \pm 37.3$ & $3.8 \pm 33.7$ \\
\hline LVESV $(\mu \mathrm{l})$ & $-3.2 \pm 4.6$ & $0.4 \pm 5.9$ & $-11.5 \pm 38.2$ & $2.8 \pm 25.5$ \\
\hline LVEF $(\%)$ & $6.8 \pm 5.1$ & $-3.5 \pm 8.7$ & $3.8 \pm 10.9$ & $-0.3 \pm 8.2$ \\
\hline
\end{tabular}

Table 3. Bland-Altman. Agreement between ECHO methods and CMR. Data are shown as mean difference \pm SD.

compared to the radial and the rotational, according to Jung et al. ${ }^{21}$ by phase contrast CMR and/or to the difficulty in the identification of endocardial borders, particularly at apical sections. The echocardiographic underestimation of LV volumes compared to CMR is already known ${ }^{22,23}$. CMR is more accurate than ECHO in tracing end-diastolic areas with its better definition of the endocardium and easier exclusion of the papillary muscles and trabeculae. This might explain the wide limits of agreement between ECHO pSAX and CMR for LV volumes and ejection fraction calculation. We wonder whether these differences are acceptable: similar results were reported in humans where the limits of agreement for ejection fraction were $-18.1-8.3 \%$ between ECHO (measured from apical 4-chamber and 2-chamber, views applying the biplane modified Simpson's method) and $\mathrm{CMR}^{23}$ and even greater in rats when measurements were taken from the $2 \mathrm{D}$ parasternal long-axis and from a single $2 \mathrm{D}$ short-axis at the mid-papillary level ${ }^{9}$.

Bland and Altman analysis confirmed that the accuracy of ECHO pSAX, compared to ECHO pLAX, was closer to CMR. Moreover, since ECHO is 3-fold faster (Fig. 3) and cheaper (Supplementary Table 1) than CMR, even using a self-gating acquisition scheme ${ }^{24}$, ECHO pSAX should be a reliable alternative for studying the LV systolic function in mice with myocardial infarction. It is clear that ECHO pSAX can be only used in anesthetized mice and echocardiographic machine must be equipped with high-resolution ultrasound and a mechanical system to move the probe in order to obtain sequential slices. As mentioned before, similar approach was recently used by O'Connor DM and colleagues ${ }^{19}$ who measured LV volumes and ejection fraction in mice with reperfused myocardial infarction using an ultrasound system, with the same resolution as we used but equipped with a customized hardware/software interface for automatic acquisition. 


\begin{tabular}{|c|c|c|}
\hline & $\begin{array}{l}\text { Inter-observer } \\
\text { variability, \% }\end{array}$ & $\begin{array}{l}\text { Intra-observer } \\
\text { variability, \% }\end{array}$ \\
\hline \multicolumn{3}{|l|}{ EDV } \\
\hline ECHO LAX SHAM & $2.3 \pm 5.9$ & $0.9 \pm 2.0$ \\
\hline ECHO SAX SHAM & $-4.2 \pm 1.4$ & $0.2 \pm 2.0$ \\
\hline CMR SHAM & $-0.2 \pm 2.6$ & $2.3 \pm 1.5$ \\
\hline \multicolumn{3}{|l|}{ ESV } \\
\hline ECHO LAX SHAM & $-4.4 \pm 6.2$ & $0.6 \pm 3.9$ \\
\hline ECHO SAX SHAM & $-0.1 \pm 8.9$ & $-0.5 \pm 2.2$ \\
\hline CMR SHAM & $-6.2 \pm 8.0$ & $1.9 \pm 3.5$ \\
\hline \multicolumn{3}{|l|}{ LVEF } \\
\hline ECHO LAX SHAM & $0.2 \pm 6.6$ & $0.7 \pm 2.6$ \\
\hline ECHO SAX SHAM & $0.4 \pm 3.6$ & $0.8 \pm 2.4$ \\
\hline CMR SHAM & $2.9 \pm 3.7$ & $-0.8 \pm 3.3$ \\
\hline \multicolumn{3}{|l|}{ EDV } \\
\hline ECHO LAX CAL & $0.3 \pm 3.1$ & $0.1 \pm 4.4$ \\
\hline ECHO SAX CAL & $3.1 \pm 3.7$ & $1.9 \pm 2.4$ \\
\hline CMR CAL & $3.7 \pm 3.15$ & $1.3 \pm 3.2$ \\
\hline \multicolumn{3}{|l|}{ ESV } \\
\hline ECHO LAX CAL & $1.2 \pm 2.5$ & $-0.4 \pm 6.8$ \\
\hline ECHO SAX CAL & $1.8 \pm 6.5$ & $1.0 \pm 3.9$ \\
\hline CMR CAL & $3.1 \pm 5.4$ & $3.1 \pm 3.4$ \\
\hline \multicolumn{3}{|l|}{ LVEF } \\
\hline ECHO LAX CAL & $2.9 \pm 7.5$ & $0.2 \pm 4.7$ \\
\hline ECHO SAX CAL & $0.28 \pm 8.2$ & $2.6 \pm 6.6$ \\
\hline CMR CAL & $2.0 \pm 6.7$ & $2.1 \pm 5.5$ \\
\hline
\end{tabular}

Table 4. Variability. Inter-observer and intra-observer variability in ECHO pLAX, ECHO pSAX and CMR for left ventricular parameters studied.

It is debated whether ECHO pLAX is a valuable method for assessing LV function in experimental models of myocardial infarction ${ }^{4}$. We found a small mean difference and acceptable $95 \%$ limits of agreement between ECHO pLAX and CMR for LV ejection fraction but not for LV volumes (Fig. 4). These results suggest that without a high-resolution ultrasound platform, the ECHO pLAX method can be used for LV ejection fraction calculation in mice with myocardial infarction, but LV volumes will be underestimated.

We found no statistical significance in the correlation between ECHO pLAX and CMR measurements in SHAM mice possible because the values were concentrated in a very narrow range (Supplementary Fig. 1).

We wondered if ECHO pSAX method could be applied to other species used as models in cardiovascular research. Two and four apical chambers views are recommended by the echocardiographic guidelines to accurately assess LV volumes and ejection fraction (particularly in asymmetric ventricles) by using the biplane method of disk summation technique ${ }^{25}$. Here we proposed the ECHO pSAX method because of the impossibility to obtain standardized and non-foreshortened apical views in mice, the experimental myocardial infarction most used animal model. Based on our experience, ECHO pSAX method could be used to evaluate rats, whereas its applicability on other species such as rabbits and feline would need further analysis. In large models like dogs and pigs, the use of two and four apical chambers views has been reported by our group and others ${ }^{26,27}$, and is fully accepted as standard method for LV volumes and ejection fraction calculation. However, our method could be an alternative to use in those animals with a non-optimal apical window.

As already mentioned, scan time during CMR may explain the higher LV volumes observed. In order to expedite CMR procedure, in the last few years, new coils with higher signal-to-noise ratios (SNR) using parallel elements and/or cryocooling techniques have been developed ${ }^{28}$. Only one study in rats ${ }^{29}$ has used a 4 -element array. Clearly due to the different cardiac dimensions between rats and mice, the SNR and the image quality obtained in that study cannot be compared with that obtained in our experimental setup. In the present study, the CMR acquisition could have been shortened by using an under sampling of the k-space; however, this procedure reduces the SNR of the image and could have produced image distortions. The prospectively triggered acquisition on EKG signal is also time-consuming. The introduction of cardiac and respiratory self-gated (Intragate ${ }^{\circledR}$ ) acquisition with retrospective reconstruction of the cardiac cycle ${ }^{24}$ could have shortened the CMR acquisition. Unfortunately, it may be optimal for assessing normal cardiac hearts but may give an inaccurate analysis in infarcted animals. First, the decrease in SNR and in the contrast-to-noise ratio between blood and myocardial wall may be challenging in the identification of the endocardial borders of the thin infarcted wall; second it may not be able to consider the possible arrhythmias and EKG disorders that can arise in CAL mice. Another issue to underline is that we saved 16 frames per cardiac cycle for accurate detection of end-diastole and end-systole phases, and this latter takes part in the total acquisition time. 


\section{Study Limitations}

In mice with severe impairment of LV function, a reduced inflow effect may lower the contrast-to-noise ratio affecting the accuracy of our measurements, as described by other authors ${ }^{30}$. However, a significant correlation was found between myocardial infarction size measured by CMR and LV volumes and ejection fraction measured by all methods studied with a positive association with LV volumes and a negative association with ejection fraction (Supplementary Fig. 2).

Since mice with large myocardial infarction are likely to be affected by repeated anesthesia ${ }^{2}$, we adopted a three days interval after ECHO before CMR to allow for the reestablishment of vital parameters and ambulatory behavior and this could have affected the actual agreement between the two imaging methods ${ }^{31}$.

We are not able to suppose whether ECHO pSAX method may improve the echocardiographic assessment of LV remodeling in different experimental models without marked variations in LV geometry (i.g hypertrophic cardiomyopathies -such as aortic banding or transgenic mice-, HFpEF models, etc). In non-dilated left ventricles, the identification of the apical end-systolic endocardial border was difficult due to the superimposition of local irregularities of the mural endocardium. We suppose that this issue is the reason why ECHO pLAX method showed better agreement with CMR.

LV mass is also an overall important variable of LV remodeling. However, several works ${ }^{32,33}$, have showed significant correlation between echocardiographic LV mass and crude LV weight in normal and post-aortic banding mice but not in myocardial infarction models. In dilated infarcted hearts, in fact, hypertrophy may be severe but may be masked by the thinning of the left ventricular wall thus the actual low LV weight may mask the presence of hypertrophic segments explaining the absence of correlation between LV mass and LV weight in myocardial infarction models. For this latter reason we did not include LV mass as variable in our study.

In the last few years hardware has made great improvements in CMR field. The new cryocoils, with their gain in the SNR, between $3.0-5.0^{34}$, have greatly reduced the CMR scanning time (namely 6 times faster) but this is still a costly hardware upgrade which is not always feasible.

\section{Conclusions}

LV volumes and ejection fraction measured by ECHO pSAX and CMR were very similar. This result has important practical implications in experimental settings, since ECHO is more readily available. However, ECHO pLAX remains a reliable method for LV function assessment of symmetrical left ventricle and for LV ejection fraction calculation in infarcted mice.

\section{References}

1. Davis, J., Maillet, M., Miano, J. M. \& Molkentin, J. D. Lost in transgenesis: a user's guide for genetically manipulating the mouse in cardiac research. Circ Res 111(6), 761-77 (2012).

2. Tremoleda, J. L., Kerton, A. \& Gsell, W. Anaesthesia and physiological monitoring during in vivo imaging of laboratory rodents: considerations on experimental outcomes and animal welfare. EJNMMI Res Aug 9, 2(1), 44 (2012).

3. Scherrer-Crosbie, M. \& Thibault, H. B. Echocardiography in translational research: of mice and men. J Am Soc Echocardiogr 21(10), 1083-92 (2008).

4. Dawson, D. et al. Quantitative 3-dimensional echocardiography for accurate and rapid cardiac phenotype characterization in mice. Circulation 110(12), 1632-7 (2004).

5. Schneider, J. E., Wiesmann, F., Lygate, C. A. \& Neubauer, S. How to perform an accurate assessment of cardiac function in mice using high-resolution magnetic resonance imaging. J Cardiovasc Magn Reson 8(5), 693-701 (2006).

6. Price, A. N. et al. Cardiovascular magnetic resonance imaging in experimental models. Open Cardiovasc Med J4, 278-92 (2010).

7. Scherrer-Crosbie, M. et al. Three-dimensional echocardiographic assessment of left ventricular wall motion abnormalities in mouse myocardial infarction. J Am Soc Echocardiogr 12(10), 834-40 (1999).

8. Amundsen, B. H. et al. A comparison of retrospectively self-gated magnetic resonance imaging and high-frequency echocardiography for characterization of left ventricular function in mice. Lab Anim 45(1), 31-7 (2011).

9. Arias, T. et al. Comparison of echocardiographic measurements of left ventricular volumes to full volume magnetic resonance imaging in normal and diseased rats. J Am Soc Echocardiogr 26(8), 910-8 (2013).

10. Rigolli, M., Anandabaskaran, S., Christiansen, J. P. \& Whalley, G. A. Bias associated with left ventricular quantification by multimodality imaging: a systematic review and meta-analysis. Open Heart Apr 27, 3(1), e000388 (2016).

11. Galli, D. et al. Mesoangioblasts, vessel-associated multipotent stem cells, repair the infarcted heart by multiple cellular mechanisms: a comparison with bone marrow progenitors, fibroblasts, and endothelial cells. Arterioscler Thomb Vasc Biol 25(4), 692-7 (2005).

12. Buser, P. T. et al. Noninvasive evaluation of global left ventricular function with use of cine nuclear magnetic resonance. $J$ Am Coll Cardiol 13(6), 1294-300 (1989).

13. Pennell, D. J. Ventricular volume and mass by CMR. J Cardiovasc Magn Reson 4(4), 507-13 (2002).

14. Lu, M. J. et al. Sample Size for Assessing Agreement between Two Methods of Measurement by Bland-Altman Method. Int J Biostat Nov 1, 12(2) (2016).

15. Altman, D. G. \& Bland, J. M. Measurement in medicine: the analysis of method comparison studies. The Statistician 32, 307-17 (1983).

16. Bland, J. M. \& Altman, D. G. Applying the right statistics: analyses of measurement studies. Ultrasound Obstet Gynecol 22(1), 85-93 (2003).

17. Sugeng, L. et al. Quantitative assessment of left ventricular size and function: side-by-side comparison of real-time threedimensional echocardiography and computed tomography with magnetic resonance reference. Circulation Aug 15, 114(7), 654-61 (2006).

18. Hoffmann, R. et al. (2014). Analysis of left ventricular volumes and function: a multicenter comparison of cardiac magnetic resonance imaging, cine ventriculography, and unenhanced and contrast-enhanced two-dimensional and three-dimensional echocardiography. J Am Soc Echocardiogr Mar, 27(3), 292-301 (2014).

19. O'Connor, D. M. et al. Heart Rate Reduction With Ivabradine Protects Against Left Ventricular Remodeling by Attenuating Infarct Expansion and Preserving Remote-Zone Contractile Function and Synchrony in a Mouse Model of Reperfused Myocardial Infarction. J Am Heart Assoc Apr 22, 5(4) (2016).

20. Bhan, A. et al. High-frequency speckle tracking echocardiography in the assessment of left ventricular function and remodeling after murine myocardial infarction. Am J Physiol Heart Circ Physiol 306, H1371-83 (2014).

21. Jung, B. et al. A quantitative comparison of regional myocardial motion in mice, rabbits and humans using in-vivo phase contrast CMR. J Cardiovasc Magn Reson Dec 27, 14, 87 (2012). 
22. Bellenger, N. G. et al. Comparison of left ventricular ejection fraction and volumes in heart failure by echocardiography, radionuclide ventriculography and cardiovascular magnetic resonance; are they interchangeable? Eur Heart J 21 (16), 1387-96 (2000).

23. Malm, S., Frigstad, S., Sagberg, E., Larsson, H. \& Skjaerpe, T. Accurate and reproducible measurement of left ventricular volume and ejection fraction by contrast echocardiography: a comparison with magnetic resonance imaging. J Am Coll Cardiol 44(5), 1030-5 (2004).

24. Hoerr, V. et al. Cardiac-respiratory self-gated cine ultra-short echo time (UTE) cardiovascular magnetic resonance for assessment of functional cardiac parameters at high magnetic fields. J Cardiovasc Magn Reson 15, 59 (2013).

25. Lang, R. M. et al. Recommendations for cardiac chamber quantification by echocardiography in adults: an update from the American Society of Echocardiography and the European Association of Cardiovascular Imaging. Eur Heart J Cardiovasc Imaging Mar; 16(3), 233-70 (2015).

26. Ristagno, G. et al. Postresuscitation treatment with argon improves early neurological recovery in a porcine model of cardiac arrest. Shock Jan; 41(1), 72-8 (2014).

27. Tidholm, A., Westling, A. B., Höglund, K., Ljungvall, I. \& Häggström, J. Comparisons of 3-,2-dimensional, and M-mode echocardiographical methods for estimation of left chamber volumes in dogs with and without acquired heart disease. J Vet Intern Med Nov-Dec, 24(6), 1414-20 (2010).

28. Niendorf, T. et al. Advancing Cardiovascular, Neurovascular, and Renal Magnetic Resonance Imaging in Small Rodents Using Cryogenic Radiofrequency Coil Technology. Front Pharmacol 6, 255 (2015).

29. Stuckey, D. J., Carr, C. A., Tyler, D. J. \& Clarke, K. Cine-MRI versus two-dimensional echocardiography to measure in vivo left ventricular function in rat heart. NMR Biomed 21, 765-72 (2008).

30. Schneider, J. E. et al. Fast, high-resolution in vivo cine magnetic resonance imaging in normal and failing mouse hearts on a vertical 11.7 T system. J Magn Reson Imaging Dec 18(6), 691-701 (2003).

31. Kohn, D. F. et al. In: Anesthesia and Analgesia in Laboratory Animals. (Academic Press U.S.A, p 157) (1997).

32. Schiller, N. B. et al. Canine left ventricular mass estimation by two-dimensional echocardiography. Circulation Jul, 68(1), 210-6 (1983).

33. Gao, X. M., Dart, A. M., Dewar, E., Jennings, G. \& Du, X. J. Serial echocardiographic assessment of left ventricular dimensions and function after myocardial infarction in mice. Cardiovasc Res Jan 14, 45(2), 330-8 (2000).

34. Wagenhaus, B. et al. Functional and morphological cardiac magnetic resonance imaging of mice using a cryogenic quadrature radiofrequency coil. PLoS One 7(8), e42383 (2012).

\section{Acknowledgements}

We are grateful to Laura Cornaghi, Alessandra Secomandi and Annarita Soldo for technical assistance and to Judith Baggott for language editing. All authors have reviewed and approved the manuscript and declare no competing interests.

\section{Author Contributions}

R.L. and L.S. conceived and designed the study; I.R., E.M. and F.F. performed experiments; I.R., M.M. and L.S. analyzed data and interpreted results of experiments; E.M., G.R. and R.L. critically discussed data; I.R. and L.S. prepared figures and wrote manuscript; I.R., E.M., F.F., M.M., G.R., R.L. and L.S. approved final version of manuscript.

\section{Additional Information}

Supplementary information accompanies this paper at https://doi.org/10.1038/s41598-019-40393-0.

Competing Interests: The authors declare no competing interests.

Publisher's note: Springer Nature remains neutral with regard to jurisdictional claims in published maps and institutional affiliations.

(c) (i) Open Access This article is licensed under a Creative Commons Attribution 4.0 International cc) License, which permits use, sharing, adaptation, distribution and reproduction in any medium or format, as long as you give appropriate credit to the original author(s) and the source, provide a link to the Creative Commons license, and indicate if changes were made. The images or other third party material in this article are included in the article's Creative Commons license, unless indicated otherwise in a credit line to the material. If material is not included in the article's Creative Commons license and your intended use is not permitted by statutory regulation or exceeds the permitted use, you will need to obtain permission directly from the copyright holder. To view a copy of this license, visit http://creativecommons.org/licenses/by/4.0/.

(C) The Author(s) 2019 